Research Paper

\title{
Solanum Incanum Extract Downregulates Aldehyde Dehydrogenase 1-Mediated Stemness and Inhibits Tumor Formation in Ovarian Cancer Cells
}

\author{
Yi-Hui Wu1, Wen-Tai Chiu2, Ming-Jer Young³, Tzu-Hao Chang, ${ }^{4}$ Yu-Fang Huang1, and Cheng-Yang \\ $\mathrm{Chou}^{1 \times}$ \\ 1. Department of Obstetrics and Gynecology, College of Medicine, National Cheng Kung University and Hospital, Tainan, Taiwan \\ 2. Department of Biomedical Engineering, National Cheng Kung University, Tainan, Taiwan \\ 3. Institute of Basic Medical Sciences, College of Medicine, National Cheng Kung University, Tainan, Taiwan \\ 4. Graduate Institute of Biomedical Informatics, Taipei Medical University, Taipei, Taiwan
}

\begin{abstract}
$\square$ Corresponding author: Cheng-Yang Chou, M.D. Department of Obstetrics and Gynecology, College of Medicine, National Cheng Kung University and Hospital, No. 138, Sheng Li Road, Tainan, 70403, Taiwan. Tel: 886 (6) 2353535 ext. 5608; Fax: 886 (6) 2766185. E-mail: chougyn@mail.ncku.edu.tw
\end{abstract}

() 2015 Ivyspring International Publisher. Reproduction is permitted for personal, noncommercial use, provided that the article is in whole, unmodified, and properly cited. See http://ivyspring.com/terms for terms and conditions.

Received: 2015.05.19; Accepted: 2015.07.20; Published: 2015.08.22

\begin{abstract}
Solanum incanum extract (SR-T100), containing the active ingredient solamargine, can induce apoptosis via upregulation of tumor necrosis factor receptor expression and activation of the mitochondrial apoptosis pathway, and has therapeutic effects in patients with actinic keratosis. Here, we evaluate the novel molecular mechanisms underlying SR-T100-regulated stemness and chemoresistance. The concentration of SR-T100 that inhibited $50 \%$ cell viability $\left(I_{50}\right)$ was lower in ovarian cancer cells than in nonmalignant cells. Furthermore, the SR-T100 IC ${ }_{50}$ in chemoresistant cells was similar to the $\mathrm{IC}_{50}$ in chemosensitive cells. Additionally, SR-T100 increased cisplatin and paclitaxel sensitivity in chemoresistant cells. SR-T100 downregulated the expression of stem cell markers, including aldehyde dehydrogenase 1 (ALDHI), Notch 1, and FoxMl, and reduced sphere formation in ovarian cancer cells. Using microarray analyses, immunoblotting, luciferase activity, and chromatin immunoprecipitation (ChIP) assays, we showed that SR-T100 suppressed the expression of $\mathrm{C} / \mathrm{EBP} \beta$ and COLI IAl, and its promoter activity, in resistant cells, but not sensitive cells. SR-T100, paclitaxel, and cisplatin inhibited the growth of A2780CP70 cells in mouse xenografts, as compared to the vehicle control, and the combination of cisplatin and SR-T100 was more effective than either treatment alone. SR-T100 may represent a potential therapeutic adjunct to chemotherapy for ovarian cancer treatment.
\end{abstract}

Key words: Solanum incanum, aldehyde dehydrogenase 1, ovarian cancer, stemness, chemoresistance

\section{Introduction}

Epithelial ovarian carcinoma (EOC) is the most lethal gynecological malignancy (1). The initial symptoms are vague, and the majority of women have advanced disease at the time of diagnosis. The standard treatment is aggressive cytoreductive surgery, followed by platinum-taxane based chemo- therapy. After therapy, a significant number of patients eventually relapse and develop chemoresistance, leading to a very low overall survival rate. Despite the incorporation of new chemotherapies for EOC treatment, only a small increase in overall survival has been achieved (2). Additionally, second-line 
chemotherapeutics can cause substantial adverse effects in patients (3). This highlights a need for novel therapeutic approaches to improve EOC treatment.

One approach to overcome this problem is the development of new agents that can be used in combination with existing chemotherapeutics to yield better results than chemotherapeutics alone (4). Accumulating evidence suggests that many natural products, including extracts and isolated chemicals, have the potential to interact with multiple targets in the signaling pathways that regulate cancer progression (5). Therefore, a systemic study of natural products is needed to define their antitumor effects and understand their mechanisms of action to develop new treatments.

The anticancer effects of Solanum species have been known for centuries (6-8). Several active ingredients, including solamargine, solasodine, and solasonine, suppress cancer growth in vitro and in vivo (7-10). Solamargine is the major glycoalkaloid in Solanum incucanum. Previous work showed solamargine increases the expression of tumor necrosis factor receptors (TNFR1 and TNFR2)(11-14) and activates the mitochondrial apoptotic pathway in human hepatocellular carcinoma (HepG2 and Hep3B), lung cancer (A549, H441, H520, H661, and H69)(15, 16), and breast cancer (SK-BR3, MCF-7, HBL-100, and ZR-75-1) cell lines $(17,18)$. Compared with paclitaxel, cisplatin, gemcitabine, and etoposide, solamargine has a superior ability to suppress human lung cancer cell growth (16). Additionally, solamargine inhibits the growth of various cultured human solid tumor cell lines, including HT-29 and HCT-15 (colon), LNCaP and PC-3 (prostate), and T47D and MDA-MB-231 (breast)(19). Recently, S. incanum extract (SR-T100), which is primarily composed of solamargine alkaloid, was shown to induce cutaneous squamous cell carcinoma apoptosis via activation of TNFRs and the mitochondrial apoptotic pathway, curing this cancer in hairless mice and actinic keratosis in human patients (20). Additionally, our preliminary findings showed that SR-T100 exhibits similar killing effects in chemoresistant cancer cells and their parental chemo-naive cells, indicating that SR-T100 may overcome chemoresistance. However, the mechanisms by which SR-T100 modulates chemoresistance remain unclear. In this study, the novel molecular mechanisms underlying SR-T100-regulated chemoresistance were elucidated, providing an understanding of its mechanism of action.

\section{Materials and Methods}

\section{Cell lines and cell culture}

The immortalized normal human ovarian sur- face epithelial cell line, IOSE-398, and human ovarian cancer cell lines ES2, TOV-21G, IGROV1, A2780, A2780CP70, ov2008, and ov2008CP20 were obtained from the American Type Culture Collection (ATCC; Manassas, VA, USA). IOSE-398 and TOV-21G cells were grown in MCDB105/M19ES2 medium with 10\% fetal calf serum (FCS). ES2 cells were grown in McCoy's 5A medium supplemented with 10\% FCS. All other cells were grown in RPMI-1640 medium with $10 \%$ FCS. Cells were grown at $37^{\circ} \mathrm{C}$ in a $5 \% \mathrm{CO}_{2}$ atmosphere. Cells were cultured and stored according to the supplier's instructions and used between passages 5 and 20. Cell lines were routinely authenticated once every 6 months through cell morphology monitoring, growth curve analysis, species verification by isoenzymology and karyotyping, identity verification using short tandem repeat profiling analysis, and contamination checks.

\section{3-(4,5-Dimethylthiazol-2-yl)-2,5-diphenyltetra zolium bromide (MTT) cytotoxicity assay}

Cell lines were cultured in 96-well flat-bottomed microtiter plates. After $72 \mathrm{~h}$ of anti-cancer drug treatment, the in vitro cytotoxic effects were determined by MTT assay at $540 \mathrm{~nm}$. The cell viability was expressed as a percentage of the control (untreated) cells (\% control).

\section{Western blot analysis}

Cells were washed in PBS, and then lysed in lysis buffer containing $50 \mathrm{mM}$ Tris- $\mathrm{HCl}$ ( $\mathrm{pH} 7.5), 150 \mathrm{mM}$ $\mathrm{NaCl}, 1 \mathrm{mM}$ EDTA, $1 \mathrm{mM} \mathrm{MgCl}$, and $0.5 \%$ Triton $\mathrm{X}-100$. Lysates were cleared by centrifugation at 13000 $\mathrm{g}$ for $20 \mathrm{~min}$ at $4^{\circ} \mathrm{C}$ and analyzed by western blot analysis. Protein samples were separated by sodium dodecyl sulfate-polyacrylamide gel electrophoresis (SDS-PAGE), transferred to a polyvinylidene difluoride membrane, and probed with the indicated antibodies. Proteins were detected by chemiluminescence.

\section{Antibodies and reagents}

Western blotting was performed using the following antibodies at the indicated dilutions: anti-aldehyde dehydrogenase 1 (ALDH1; 1:1000; BD Biosciences; Franklin Lakes, NJ, USA), anti-Notch1 (1:1000), anti-COL11A1 (1:1000; Abcam; Cambridge, UK), anti-FoxM1 (1:1000; Santa Cruz Biotechnology; Santa Cruz, CA, USA), and anti- $\beta$ actin (1:5000; Sigma; St. Louis, MO, USA). Cisplatin (Fresenius Kabi Oncology Ltd, Haryana, India) and paclitaxel (Corden Pharma Latina S.P.A., Sermoneta, Italy) were kindly provided by the Cancer Center at National Cheng Kung University Hospital, Tainan, Taiwan. SR-T100 isolated from $S$. incanum was provided by G\&E Herbal Biotechnology Company (Tainan, Taiwan). 


\section{Sphere formation assay}

Standard sphere formation assays were performed according to Zhang et al. (21) with minor modifications. Cells $\left(1 \times 10^{3}\right)$ were resuspended in serum-free DMEM/F12 medium supplemented with $5 \mu \mathrm{g} / \mathrm{mL}$ insulin (Sigma), $20 \mathrm{ng} / \mathrm{mL}$ human recombinant epidermal growth factor (EGF; Invitrogen, Life Technologies, Carlsbad, CA, USA), and $10 \mathrm{ng} / \mathrm{mL}$ basic fibroblast growth factor (Invitrogen) in ultra-low attachment plates (Corning Costar, Corning, NY, USA). Spheres that arose within 1-2 weeks were counted. Colony diameters $>50 \mu \mathrm{m}$ were counted as a single positive colony. The middle field was chosen to count spheres, and two fields for each plate were counted under a dissecting microscope. For all sphere formation experiments, a minimum of eight wells were run for each condition. All data represent the mean \pm SEM of three separate experiments and at least 24 different fields.

\section{Microarray analysis and data processing}

Total RNA was isolated using the RNeasy Micro Kit (Qiagen; Valencia, CA, USA), quantified, and checked for quality with a Bioanalyzer 2100 system (Agilent; Palo Alto, CA, USA). Labeled probes for Affymetrix DNA microarray analysis were prepared according to the manufacturer's instructions. Biotin-labeled cRNA, produced by in vitro transcription, was fragmented and hybridized to Affymetrix U133A GeneChip arrays (22,283 probe sets, Santa Clara, CA, USA) at $45^{\circ} \mathrm{C}$ for $16 \mathrm{~h}$, and then washed and stained using GeneChip Fluidics. The arrays were scanned to a target intensity of 500 by a GeneArray Scanner, and patterns of hybridization were detected as light emitted from the fluorescent reporter groups incorporated into the target and hybridized to oligonucleotide probes. Image acquisition and probe quantification was performed using Affymetrix GeneChip Operating Software. Microarray quality control was performed using $\mathrm{R}$ package affyQCReport software (22). The gcrma function in the R package affy was applied to normalize the CEL files using the RMA method (23). Data are MIAME compliant and have been deposited in the Gene Expression Omnibus.

\section{Plasmid construction and site-directed muta- genesis}

The COL11A1 polymerase chain reaction product was cloned into the $K p n \mathrm{I}$ and $\mathrm{XhoI}$ sites in the pGL4 vector. The resulting construct was confirmed by DNA sequencing. The COL11A1 promoter deletion construct, COL11A1-541/-203, was similarly generated using the COL11A1-541/+1 construct as a template.

\section{Luciferase reporter assay}

Luciferase assays were conducted using a luciferase reporter assay system (Promega; Madison, WI, USA) $48 \mathrm{~h}$ after transfection. Normalized luciferase activity is reported as luciferase activity $/ \beta$-galactosidase activity.

\section{Chromatin immunoprecipitation (ChIP) assay}

Native protein-DNA complexes were cross-linked by treatment with $1 \%$ formaldehyde for $15 \mathrm{~min}$. The ChIP assay was performed as previously reported (24). Briefly, equal aliquots of isolated chromatin were subjected to immunoprecipitation with anti-c/EBP $\beta$ and IgG monoclonal antibodies.

\section{Xenograft animal model}

All animal procedures were reviewed and approved by the Institutional Animal Care and Use Committee at National Cheng Kung University (No. 102267). Female 6-week-old NOD-SCID mice (Charles River Laboratories) were subcutaneously implanted in the flank with A2780CP70 cells $\left(1 \times 10^{6}\right.$ cells/100 $\mu \mathrm{L})$. Tumor dimensions were measured 2 to 3 times per week, and volume was calculated as length $\times$ width $\times$ height $\times 0.52$. Once tumors reached $20 \mathrm{~mm}^{3}$, the mice were randomly assigned to 10 groups $(n=6)$. Animals in each group received the same volume of saline, cisplatin, or SR-T100 in $200 \mu \mathrm{L}$ by intraperitoneal injection. Treatment frequency was 1 time per day for SR-T100 and 1 time per 3 days for cisplatin. Tumor growth, tumor imaging, and body weights were determined, as described (25). The mice were sacrificed by $\mathrm{CO}_{2}$ inhalation and xenograft tumor tissues were excised.

\section{Statistical analysis}

Statistical analysis was performed using the Statistical Package for the Social Sciences software, version 17.0, for Windows (SPSS Inc.). Frequency distributions between categorical variables were compared using the Pearson chi-square test and Fisher's exact method. Student's $t$-test and Mann-Whitney $U$ test were used to compare two groups with normally and non-normally distributed interval data, respectively. One-way ANOVA and Kruskal-Wallis tests were used compare three or more groups with normally distributed and non-normally distributed interval data, respectively.

\section{Results}

\section{SR-T100 sensitizes chemoresistant cells to cisplatin and paclitaxel}

To illustrate the anticancer effects of SR-T100 in ovarian cancer, we first evaluated SR-T100-induced cytotoxicity using the MTT assay in ovarian cancer 
cells and IOSE-398 cells. As shown in Fig. 1A, the concentration of SR-T100 that reduced cell viability $50 \%\left(\mathrm{IC}_{50}\right)$ was lower in malignant cells $\left(\mathrm{IC}_{50}\right.$ of ES2 $=$ $12.83 \mu \mathrm{g} / \mathrm{ml} ; \mathrm{IC}_{50}$ of TOV-21G $=14.88 \mu \mathrm{g} / \mathrm{ml} ; \mathrm{IC}_{50}$ of IGROV1 $=14.69 \mu \mathrm{g} / \mathrm{ml})$ than in nonmalignant cells $\left(\mathrm{IC}_{50}\right.$ of IOSE-398 $=27.73 \mu \mathrm{g} / \mathrm{ml}$ ) (Fig. 1A), suggesting that SR-T100 is less toxic in IOSE-398 cells. The effect of SR-T100 on chemosensitivity was examined in the following paired ovarian cancer cell lines: A2780 and A2780CP70, and ov2008 and ov2008CP20. Chemoresistant cells were less sensitive to cisplatin than chemosensitive cells (Fig. 1B). The $\mathrm{IC}_{50}$ values for SR-T100 treatment in resistant cells $\left(\mathrm{IC}_{50}\right.$ of
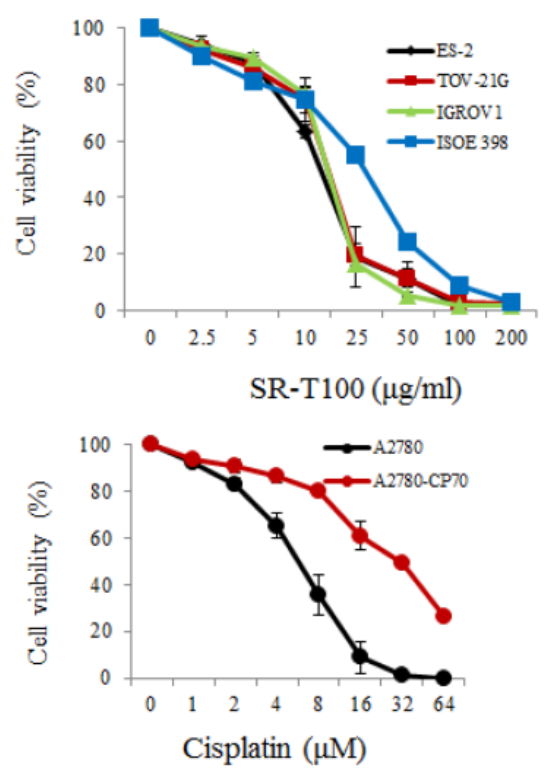

C
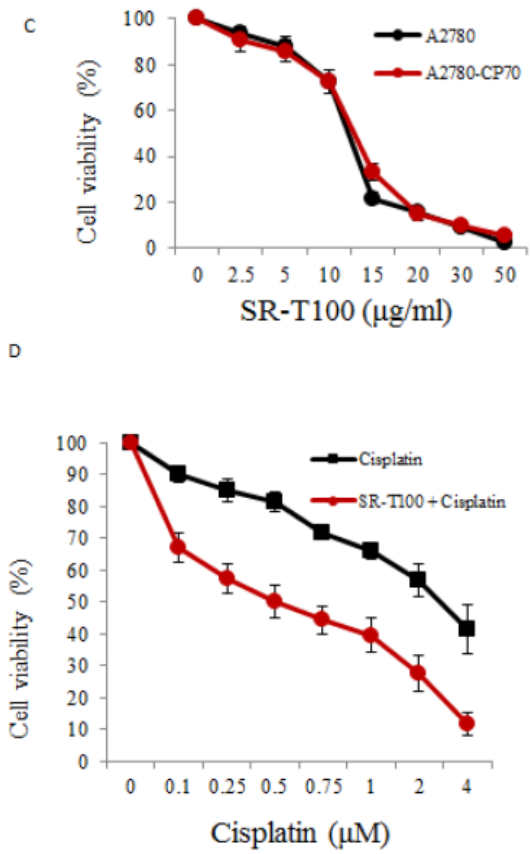

$\mathrm{A} 2780 \mathrm{CP} 70=12.62 \mu \mathrm{g} / \mathrm{ml} ; \mathrm{IC}_{50}$ of ov2008CP20 $=15.94$ $\mu \mathrm{g} / \mathrm{ml}$ ) were similar to those in sensitive cells $\left(\mathrm{IC}_{50}\right.$ of A2780 $=11.91 \mu \mathrm{g} / \mathrm{ml} ; \mathrm{IC}_{50}$ of ov2008 $=15.25 \mu \mathrm{g} / \mathrm{ml}$ ) (Fig. 1C). These results suggest that SR-T100 is cytotoxic in both chemosensitive and chemoresistant ovarian cancer cells. More importantly, the $\mathrm{IC}_{50}$ values in TOV-21G cells decreased after treatment with SR-T100 in combination with various concentrations of cisplatin or paclitaxel, as compared with cisplatin or paclitaxel treatment alone (Fig. 1D). These results suggest that SR-T100 sensitizes chemoresistant cells to cisplatin and paclitaxel.
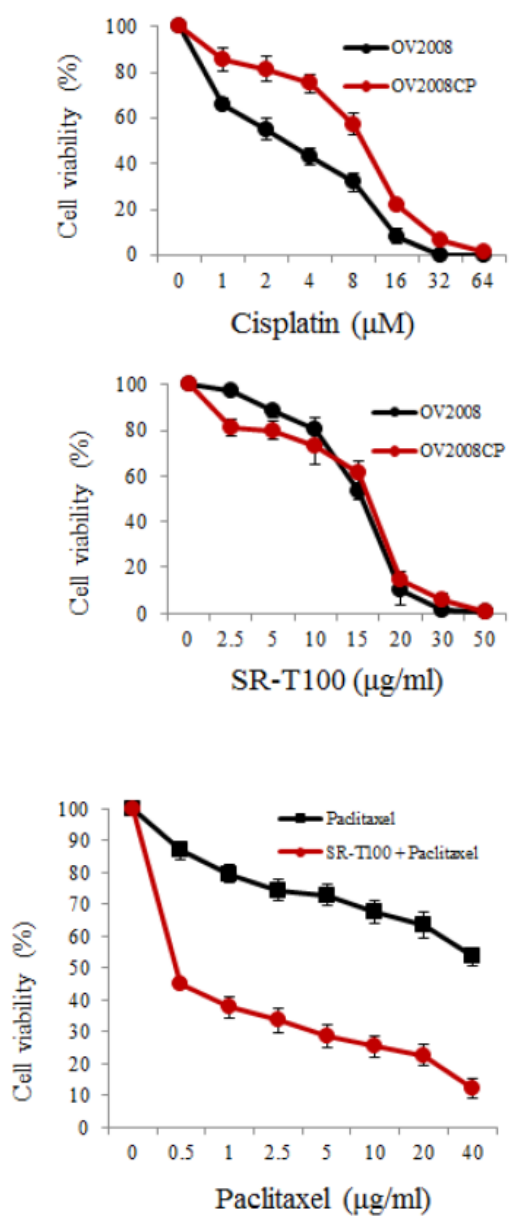

Figure 1. SR-T100 treatment increases cell sensitivity to cisplatin and paclitaxel. Cell viability following SR-T100 (A), cisplatin (B), or combined (C) treatment was measured by MTT assay. (D) Cell viability following combination treatment with SR-T100 and cisplatin or paclitaxel was measured by MTT assay. All experiments were performed in triplicate. 


\section{SR-T100 inhibits stem cell marker expression and sphere formation in ovarian cancer cells}

Recent studies have shown that subpopulations of cancer cells, cancer stem-like cells (CSCs), are characterized by their enhanced tumor formation ability and drug resistance. CSCs share some markers with stem cells, including ALDH1 and Notch1 (21, 26, 27). Additionally, our recent work has indicated that FoxM1 regulates epithelial-mesenchymal transition, stemness, and chemoresistance in epithelial ovarian carcinoma cells (28). Thus we evaluated whether ALDH1, Notch1, and FoxM1 were inhibited by SR-T100. As shown in Figure 2A, SR-T100 dose-dependently downregulated the expression of ALDH1, Notch1, and FoxM1 in chemoresistant A2780CP70 cells. This decreased expression was not observed in chemosensitive A2780 cells. Sphere formation in A2780CP70 cells was also dose-dependently inhibited by SR-T100 treatment (Fig. 2B).

\section{Downregulation of $\mathrm{c} / \mathrm{EBP} \beta$ and COL11A1 lev- els by SR-T100 in chemoresistant ovarian cancer cells by expression profiling}

To identify the genes regulated by SR-T100, A2780CP70 cells were treated with 2.5 or $5 \mu \mathrm{g} / \mathrm{ml}$ SR-T100, and a gene expression array was performed.
Microarray analysis showed that 197 genes (66 upregulated and 131 downregulated) were differentially expressed between SR-T100-treated and control cells (Tables 1 and 2). Among these genes, c/EBP $\beta$ and COL11A1 were among the most downregulated genes. Our recent findings show that COL11A1 plays a dual role in EOC tumor progression and chemoresistance, and that the $c / E B P \beta$ binding site on the COL11A1 promoter is a major determinant of cisplatin- and paclitaxel-dependent COL11A1 activation (29). Our results showed that the expression of c/EBP $\beta$ and COL11A1 was enhanced by cisplatin, and that this elevated expression was inhibited by SR-T100 in chemoresistant A2780CP70 cells. In contrast, the expression level of c/EBP $\beta$ and COL11A1 was largely unaffected in chemosensitive A2780 cells by either cisplatin or SR-T100 treatment (Fig. 3A). In addition, the increased promoter activity by cisplatin in the region between -541 and -203 on COL11A1, which is important for the transcriptional regulation by cisplatin and paclitaxel (29), was decreased in SR-T100-treated A2780CP70 cells, but not in A2780 cells (Fig. 3B). ChIP assays further indicated that the binding of $\mathrm{c} / \mathrm{EBP} \beta$ to the COL11A1 promoter was inhibited by SR-T100 alone and SR-T100 plus cisplatin (Fig. 3C).

A
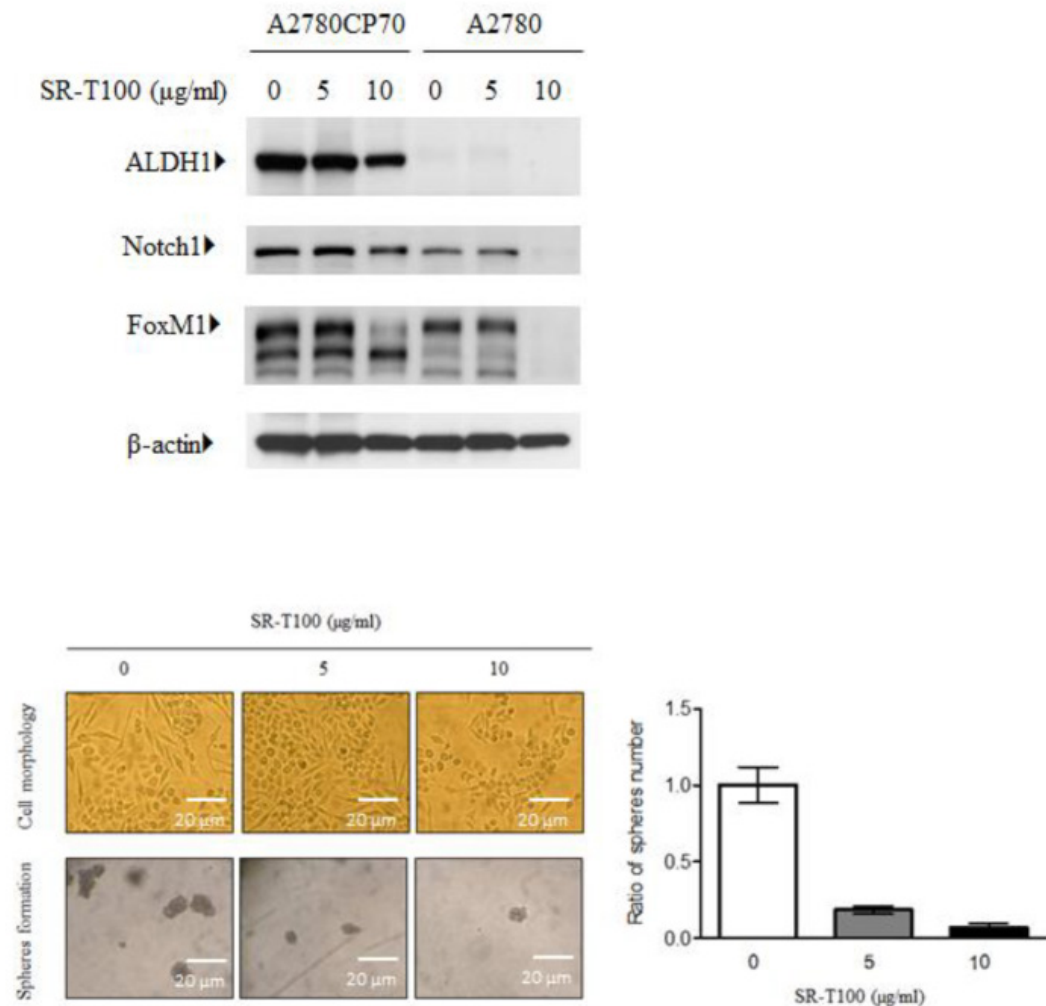

Figure 2. SR-T1 00 treatment reduces stem cells marker expression and sphere formation in chemoresistant ovarian cancer cells. (A) Cells were treated with SR-T100 (2.5 or $5 \mu \mathrm{g} / \mathrm{ml})$ for $48 \mathrm{~h}$, and cell lysates were collected for western blotting. (B) Cells were treated with SR-T100 (2.5 or $5 \mu \mathrm{g} / \mathrm{ml})$ and were evaluated by sphere formation assays. Representative images show spheres generated from single-cell cultures after 10 days. The lower panel depicts the relative sphere formation ratio (scale bar $=200 \mu \mathrm{m})$. 
A

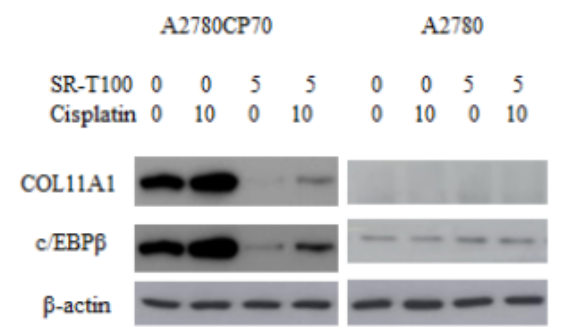

B

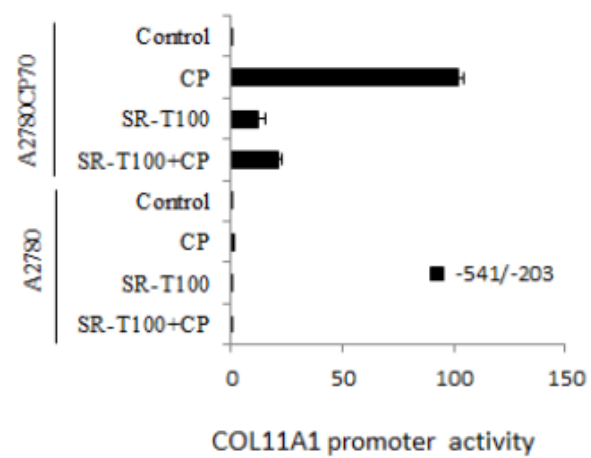

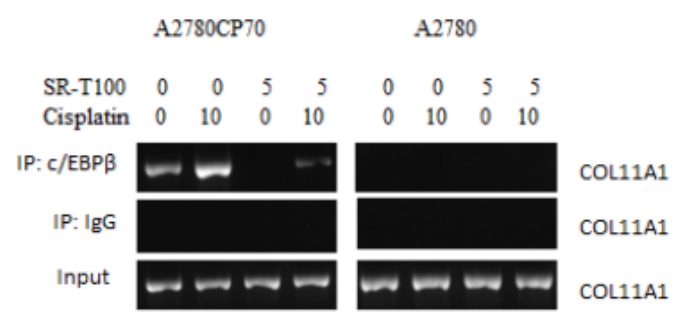

Figure 3. SR-T1 100 downregulated c/EBP $\beta$ and COL1 1 Al in chemoresistant ovarian cancer cells. (A) Left panel: Cells were treated with SR-T100 (2.5 or $5 \mu \mathrm{g} / \mathrm{ml})$ for $48 \mathrm{~h}$, and cell lysates were collected for western blotting. Right panel: COL1 IAl and c/EBP $\beta$ protein expression in cells treated with SR-T100 (5 $\mu \mathrm{g} / \mathrm{ml})$ and cisplatin $(10 \mu \mathrm{M})$ for 48 days. (B) A2780CP70 cells transfected with the indicated COL11A1 promoter constructs were treated with SR-T100 and cisplatin for 48 h. The luciferase activity was measured and normalized to Renilla luciferase activity. All experiments were performed in triplicate. (C) A ChIP assay was performed to evaluate the binding of $\mathrm{c} / \mathrm{EBP} \beta$ to the COL11A1 promoter after treatment of A2780CP70 and A2780 cells for $48 \mathrm{~h}$ with varying cisplatin concentrations.

Table 1. The 66 genes upregulated in response to SR-T100 treatment in A2780CP70 cells.

\begin{tabular}{|l|l|l|l|}
\hline Gene & Log2 ratio & Gene & Log2 ratio \\
\hline HSPA1B & 2.94 & FABP5L2 & 1.22 \\
\hline LOC399988 & 2.48 & M6PRBP1 & 1.22 \\
\hline HMOX1 & 2.26 & C7ORF68 & 1.22 \\
\hline VGF & 2.15 & GNPDA1 & 1.21 \\
\hline HSPA8 & 1.99 & FEN1 & 1.20 \\
\hline TFRC & 1.94 & DNAJA1 & 1.20 \\
\hline SNORA61 & 1.76 & B4GALT5 & 1.20 \\
\hline RPL29 & 1.72 & RAB7L1 & 1.19 \\
\hline ID3 & 1.63 & MND1 & 1.19 \\
\hline TMEM158 & 1.54 & SLC35B2 & 1.19 \\
\hline C18ORF55 & 1.52 & RPL36A & 1.18 \\
\hline HSD17B1 & 1.51 & LOC85389 & 1.17 \\
\hline MED31 & 1.49 & CKS2 & 1.17 \\
\hline ELOF1 & 1.48 & TAGLN & 1.15 \\
\hline MAD2L1 & 1.43 & MCM10 & 1.14 \\
\hline LOC100129828 & 1.41 & LOC643387 & 1.14 \\
\hline MRPL36 & 1.38 & MRPL34 & 1.13 \\
\hline FHL2 & 1.38 & NTS & 1.13 \\
\hline C6ORF136 & 1.38 & UTP11L & 1.11 \\
\hline CCDC58 & 1.36 & LOC653506 & 1.09 \\
\hline SF3A3 & 1.34 & MFSD5 & 1.09 \\
\hline CCDC56 & 1.31 & CDC25A & 1.09 \\
\hline ITPKA & 1.31 & C7ORF30 & 1.08 \\
\hline TUBB2C & 1.29 & RABGGTB & 1.07 \\
\hline SERPINE1 & 1.27 & TSPAN9 & 1.06 \\
\hline UCRC & 1.27 & LRRC20 & 1.05 \\
\hline SPHK1 & 1.26 & ZC3HAV1 & 1.05 \\
\hline C6ORF115 & 1.26 & MAGOH & 1.04 \\
\hline LSM3 & 1.26 & TM4SF4 & 1.03 \\
\hline AP1S1 & 1.25 & EIF4E2 & 1.03 \\
\hline C17ORF79 & 1.24 & RPL34 & 1.01 \\
\hline FLJ35767 & 1.23 & FAM103A1 & 1.00 \\
\hline MMP1 & 1.23 & UNG & 1.00 \\
\hline & & & \\
\hline & & & \\
\hline
\end{tabular}

\section{SR-T100 increases cisplatin sensitivity in mouse xenografts}

To determine whether SR-T100 could suppress tumor growth, mice were injected subcutaneously with $1 \times 10^{6} \mathrm{~A} 2780 \mathrm{CP} 70$ cells and treated with intraperitoneal injection of SR-T100, with or without adding cisplatin (Figs. 4A and 4B) or paclitaxel (Figs. 4D and $4 \mathrm{E}$ ). When compared to the vehicle controls, single treatment with cisplatin $(P=0.002)$, varying SR-T100 doses (SR-T100 $2.5 \mathrm{mg} / \mathrm{kg}$, P = 0.003; SR-T100 $5.0 \mathrm{mg} / \mathrm{kg}, \mathrm{P}=0.002$ ), or paclitaxel (paclitaxel 2.5 $\mathrm{mg} / \mathrm{kg}, \mathrm{P}=0.001$; paclitaxel $5.0 \mathrm{mg} / \mathrm{kg}, \mathrm{P}=0.001$; paclitaxel $10 \mathrm{mg} / \mathrm{kg}, \mathrm{P}=0.001$ ) significantly inhibited the growth of A2780CP70 cells in mouse xenografts. Moreover, combinations of cisplatin and SR-T100 (SR-T100 $2.5 \mathrm{mg} / \mathrm{kg}+$ cisplatin $3 \mathrm{mg} / \mathrm{kg}$ vs. controls, $\mathrm{P}=0.002 ;$ SR-T100 $5 \mathrm{mg} / \mathrm{kg}+$ cisplatin $3 \mathrm{mg} / \mathrm{kg}$ vs. controls, $\mathrm{P}=0.002$ ) significantly reduced tumor volumes, as compared to the controls. The tumor suppression effect was greater with combination treatment (SR-T100 $5 \mathrm{mg} / \mathrm{kg}$ and cisplatin $3 \mathrm{mg} / \mathrm{kg}$ ) than with SR-T100 alone $(5 \mathrm{mg} / \mathrm{kg}$ alone; $\mathrm{P}=0.046)$. An effect was also found between the combination group and cisplatin alone group $(P=0.032)$. However, SR-T100 did not significantly increase the sensitivity to paclitaxel in chemoresistant cells (Fig. 4D). The body weight of animals receiving cisplatin, paclitaxel, 
SR-T100, or combined treatment remained relatively unaltered, suggesting negligible toxicity was observed (Figs. 4C and 4F). These results further confirmed that SR-T100 has the potential to kill chemoresistant cancer cells.

Table 2. The 131 genes downregulated in response to SR-T100 treatment in A2780CP70 cells.

\begin{tabular}{|c|c|c|c|}
\hline Gene & Log2 ratio & Gene & Log2 ratio \\
\hline FGF21 & 2.94 & C1ORF24 & 1.57 \\
\hline DDIT4 & 2.92 & $\begin{array}{l}\text { DDIT3 } \\
\end{array}$ & 1.55 \\
\hline ATF3 & 2.72 & ZNF627 & 1.55 \\
\hline INHBE & 2.64 & ITGAV & 1.55 \\
\hline TXNIP & 2.29 & SLC39A14 & 1.54 \\
\hline TSC22D3 & 2.13 & LOC727758 & 1.54 \\
\hline SLC38A2 & 2.06 & IRF2BP2 & 1.53 \\
\hline ARHGEF2 & 2.04 & WARS & 1.52 \\
\hline FOXO3 & 2.03 & CBLB & 1.52 \\
\hline DDR2 & 2.03 & SLC6A9 & 1.51 \\
\hline PCK2 & 2.01 & TUBE1 & 1.51 \\
\hline ASNS & 1.90 & SLC38A1 & 1.50 \\
\hline CEBPB & 1.86 & MUC15 & 1.50 \\
\hline HIF0 & 1.84 & COL5A2 & 1.49 \\
\hline IFIT2 & 1.79 & TRIB3 & 1.49 \\
\hline LOC729779 & 1.79 & $\begin{array}{l}\text { TNFRSF19 } \\
\end{array}$ & 1.49 \\
\hline SLC7A5 & 1.75 & SLC7A1 & 1.47 \\
\hline ITGA5 & 1.73 & CHD9 & 1.46 \\
\hline IL21R & 1.71 & HOXC6 & 1.46 \\
\hline NT5C2 & 1.71 & PABPC1L & 1.45 \\
\hline CLIC4 & 1.71 & GARS & 1.45 \\
\hline PSPH & 1.70 & CLDN1 & 1.45 \\
\hline PDE4D & 1.70 & RCOR2 & 1.43 \\
\hline CGGBP1 & 1.69 & PRKDC & 1.43 \\
\hline ADM2 & 1.69 & CEBPG & 1.43 \\
\hline SSX21P & 1.68 & TCEA1 & 1.42 \\
\hline FAM129A & 1.66 & UHRF1BP1 & 1.42 \\
\hline BTG1 & 1.66 & HNRPDL & 1.41 \\
\hline PSAT1 & 1.63 & LOC653103 & 1.39 \\
\hline COL11A1 & 1.63 & TNFRSF10B & 1.38 \\
\hline CALCRL & 1.62 & GPT2 & 1.37 \\
\hline CNTNAP1 & 1.62 & LRBA & 1.37 \\
\hline PPP1R15A & 1.57 & AARS & 1.37 \\
\hline EV15L & 1.37 & KCTD3 & 1.17 \\
\hline TTC39B & 1.36 & SESN2 & 1.17 \\
\hline LONP1 & 1.36 & SERPINF1 & 1.16 \\
\hline SREBF1 & 1.35 & SMARCA1 & 1.16 \\
\hline MTHFD2 & 1.35 & TRUB1 & 1.16 \\
\hline PHGDH & 1.34 & SARS & 1.15 \\
\hline ULK1 & 1.34 & VEZT & 1.14 \\
\hline EPRS & 1.34 & XBP1 & 1.14 \\
\hline CALD1 & 1.33 & MLLT10 & 1.14 \\
\hline LOC732432 & 1.33 & KDM5B & 1.12 \\
\hline SPRY2 & 1.30 & ZZZ3 & 1.12 \\
\hline CNOT1 & 1.30 & ZCCHC8 & 1.12 \\
\hline FBXO11 & 1.29 & YY1AP1 & 1.11 \\
\hline KLF9 & 1.29 & RAS1P1 & 1.09 \\
\hline GRPEL2 & 1.29 & TNFSF18 & 1.09 \\
\hline ULBP1 & 1.28 & SCRN1 & 1.09 \\
\hline GPR126 & 1.28 & CHAC1 & 1.08 \\
\hline COL3A1 & 1.26 & PLK2 & 1.08 \\
\hline LOC440341 & 1.23 & BNIP2 & 1.08 \\
\hline LOC728734 & 1.23 & SERINC1 & 1.06 \\
\hline HERPUD1 & 1.22 & ZFP90 & 1.05 \\
\hline C16ORF58 & 1.22 & ETV5 & 1.05 \\
\hline STARD4 & 1.22 & FASN & 1.05 \\
\hline ZNF643 & 1.22 & UBR4 & 1.05 \\
\hline TSC22D1 & 1.22 & AKNA & 1.04 \\
\hline C14ORF4 & 1.21 & ACLY & 1.04 \\
\hline SEZ6L2 & 1.20 & 1ARS & 1.04 \\
\hline ERRF11 & 1.19 & BRSK1 & 1.03 \\
\hline RAD21 & 1.18 & ABCA1 & 1.02 \\
\hline SLC3A2 & 1.18 & KATNB1 & 1.02 \\
\hline CTDSP2 & 1.18 & SLC1A5 & 1.02 \\
\hline ZNF827 & 1.18 & DFNA5 & 1.01 \\
\hline LOC440353 & 1.17 & LOC650215 & 1.01 \\
\hline DYNC1H1 & 1.17 & & \\
\hline
\end{tabular}

\section{Discussion}

Solamargine effectively induces apoptosis in various cancer cell lines. In contrast, similar concentrations of solamargine did not cause apoptosis in normal cells, such as bone marrow cells, fibroblasts, and normal hepatocytes, suggesting it preferentially kills malignant cells $(13-18,30)$. Previous studies indicate that solamargine promotes cell death via the activation of TNFRs (11-14) and the mitochondrial apoptotic pathway (15-18). Recently, SR-T100 was shown to induce cutaneous squamous cell carcinoma cells apoptosis via similar mechanisms and suppresses tumor growth in mouse xenografts and patients with actinic keratosis (20). In this study, we reveal the novel molecular mechanism underlying SR-T100-induced cytotoxicity, via suppression of ALDH1-mediated stemness and COL11A1-regulated chemoresistance, thereby suppressing tumour growth in ovarian cancer cells.

Recent studies have shown that CSCs, which are characterised by enhanced tumour formation ability and drug resistance, share some stem cell markers, including ALDH1 and Notch1 (21, 26, 27). Our recent report demonstrates that ALDH1 directly regulates the functions of ovarian cancer cells. ALDH1 expression is closely associated with tumorigenic potential in ovarian cancer cell lines, and FoxM1 and Notch1 are important downstream effectors required for ALDH1-induced cancer stemness (31). Additionally, we further showed that FoxM1 regulates epithelial-mesenchymal transition, stemness, and chemoresistance in epithelial ovarian carcinoma cells (28). In the present study, SR-T100 inhibited CSC marker and FoxM1 expression and sphere formation in ovarian cancer cells. These results indicate that SR-T100 exerts killing effects in chemoresistant ovarian cancer cells and suppresses tumor growth in mouse xenografts.

Chemoresistance often causes patient death, due to a lack of effective treatments. Li et al. (32) reported that solamargine effectively triggers apoptosis in multidrug-resistant tumor cells, which is associated with actin disruption and downregulation of multiple drug resistance 1 expression. In addition to reduced stemness, we showed that SR-T100 regulates cell sensitivity to anticancer drugs via downregulation of $c / E B P \beta$ and COL11A1. Our recently report demonstrates that the activation of COL11A1 in ovarian cancer cells by cisplatin and paclitaxel confers chemoresistance by activating the Akt/c/EBP $\beta$ pathway and stabilizing phosphoinositide-dependent kinase-1 (29). In the present study, we provided the first evidence that SR-T100 enhances cell sensitivity to cisplatin and paclitaxel by downregulating the binding activity of c/EBP $\beta$ to COL11A1 promoter. 

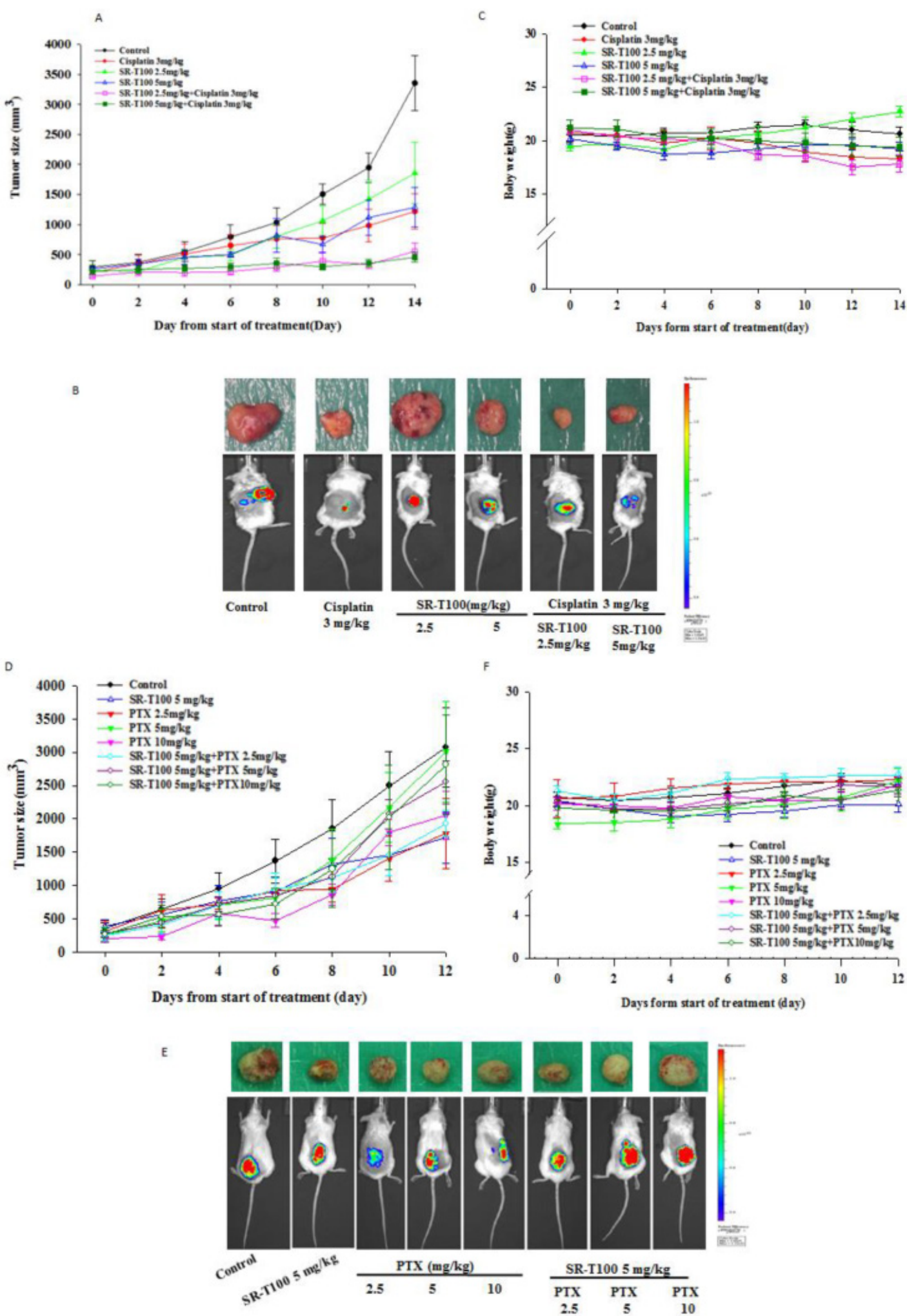

Figure 4. SR-T100 increases mouse xenograft sensitivity to cisplatin. (A) Tumor volumes in A2780CP70 cells. (B) Representative tumor volumes in A2780CP70 cells. (C) Body weight of animals following SR-T100 treatment. PTX: paclitaxel.

In addition to inducing apoptosis, SR-T100 has been shown to sensitize breast cancer cells to cisplatin and epirubicin (18). Liang et al. (16) reported that combination therapy using low concentrations of solamargine and the low-toxicity topoisomerase II inhibitor epirubicin synergistically enhanced cytotoxicity in non-small cell lung cancer cells. In the present study, SR-T100 increased the sensitivity of chemoresistant ovarian cancer cells to cisplatin and paclitaxel in vitro. Furthermore, combination treat- 
ment using cisplatin and SR-T100 was more effective in inhibiting the growth of A2780CP70 cells in mouse xenografts than either therapeutic alone. Taken together, these results suggest that SR-T100 can enhance cell sensitivity to anticancer drugs and may have potential against chemoresistant ovarian cancer cells.

\section{Acknowledgments}

SR-T100 was a kind gift from Professor Kou-Wha Kuo, Research Center of Herbal Medicines, G\&E Herbal Biotechnology, National Tainan Science Park, Tainan 744, Taiwan. This work was supported by the National Science Council under Grant Number: NSC 101-2325-B-006-022, 102-2314-B-006-081, and MOST 103-2314-B-006-069. This study was also supported by the Department of Health under Grant Number: 102TM1050, and in part by the Headquarters of University Advancement at the National Cheng Kung University, which is sponsored by the Ministry of Education, Taiwan, ROC.

\section{Author Contributions}

Conceived and designed the experiments: YHW and CYC. Performed the experiments: YHW, WTC and MJY. Analyzed the data: THC and YFH. Wrote the paper: YHW and CYC.

\section{Competing Interests}

The authors have declared that no competing interest exists.

\section{References}

1. Siegel R, Naishadham D, Jemal A. Cancer statistics, 2012. Cancer J Clin 2012; 62: $10-29$.

2. Cho KR and Shih IM. Ovarian cancer. Annu Rev Pathol 2009; 4: 287-313.

3. Heidegger I, Massoner P, Eder IE, et al. Novel therapeutic approaches for the treatment of castration-resistant prostate cancer. J Steroid Biochem Mol Biol 2013; 138C: 248-256.

4. Alberti C. Taxane- and epothilone-based chemotherapy: from molecule cargo cytoskeletal logistics to management of castration-resistant prostate carcinoma. Eur Rev Med Pharmacol Sci 2013; 17: 1658-1664.

5. Meiyanto E, Hermawan A, Anindyajati. Natural products for cancer-targeted therapy: citrus flavonoids as potent chemopreventive agents. Asian Pac J Cancer Prev 2012; 13: 427-436.

6. Cham BE, Gilliver M, Wilson L. Antitumour effects of glycoalkaloids isolated from Solanum sodomaeum. Planta Med 1987; 53: 34-36.

7. Cham BE, Meares HM. Glycoalkaloids from Solanum sodomaeum are effective in the treatment of skin cancers in man. Cancer Lett 1987; 36: 111-118.

8. Kupchan SM, Barboutis SJ, Knox JR, Cam CA. Beta-solamarine: tumor inhibitor isolated from Solanum dulcamara. Science 1965; 150: 1827-1828.

9. Cham BE, Daunter B. Solasodine glycosides. Selective cytotoxicity for cancer cells and inhibition of cytotoxicity by rhamnose in mice with sarcoma 180 . Cancer Lett 1990; 55: 221-225.

10. Lee KR, Kozukue N, Han JS, et al. Glycoalkaloids and metabolites inhibit the growth of human colon (HT29) and liver (HepG2) cancer cells. J Agric Food Chem 2004; 52: 2832-2839.

11. Liang CH, Shiu LY, Chang LC, Sheu HM, Kuo KW. Solamargine upregulation of Fas, downregulation of HER2, and enhancement of cytotoxicity using epirubicin in NSCLC cells. Mol Nutr Food Res 2007; 51: 999-1005.

12. Chiu HF, Lin CC, Yang CC, Yang F. The pharmacological and pathological studies on several hepatic protective crude drugs from Taiwan (II). Am J Chin Med 1989; 17: 17-23.

13. Chang LC, Tsai TR, Wang JJ, Lin CN, Kuo KW. The rhamnose moiety of solamargine plays a crucial role in triggering cell death by apoptosis. Biochem Biophys Res Commun 1998; 242: 21-25.

14. Hsu SH, Tsai TR, Lin CN, Yen MH, Kuo KW. Solamargine purified from Solanum incanum Chinese herb triggers gene expression of human TNFR I which may lead to cell apoptosis. Biochem Biophys Res Commun 1996; 229: $1-5$.

15. Kuo KW, Hsu SH, Li YP, et al. Anticancer activity evaluation of the solanum glycoalkaloid solamargine. Triggering apoptosis in human hepatoma cells. Biochem Pharmacol 2000; 60: 1865-1873.

16. Liang CH, Liu LF, Shiu LY, Huang YS, Chang LC, Kuo KW. Action of solamargine on TNFs and cisplatin-resistant human lung cancer cells. Biochem Biophys Res Commun 2004; 322: 751-758.

17. Liu LF, Liang CH, Shiu LY, Lin WL, Lin CC, Kuo KW. Action of solamargine on human lung cancer cells - enhancement of the susceptibility of cancer cells to TNFs. FEBS Lett 2004; 577: 67-74.

18. Shiu LY, Chang LC, Liang CH, Huang YS, Sheu HM, Kuo KW. Solamargine induces apoptosis and sensitizes breast cancer cells to cisplatin. Food Chem Toxicol 2007; 45: 2155-2164.

19. Hu K, Kobayashi H, Dong A, Jing Y, Iwasaki S, Yao X. Antineoplastic agents. III. Steroidal glycosides from Solanum nigrum. Planta Med 1999; 65: 35-38.

20. Wu CH, Liang $\mathrm{CH}$, Shiu LY, et al. Solanum incanum extract (SR-T100) induces human cutaneous squamous cell carcinoma apoptosis through modulating tumor necrosis factor receptor signaling pathway. J Dermatol Sci 2011; 63: 83-92.

21. Zhang S, Balch C, Chan MW, et al. Identification and characterization of ovarian cancer-initiating cells from primary human tumors. Cancer Res 2008; 68: 4311-4320.

22. Gentleman C, et al. R. affyQCReport: QC Report Generation for affyBatch objects. 2005.

23. Gautier L, Cope L, Bolstad BM, Irizarry RA. Affy-analysis of Affymetrix GeneChip data at the probe level. Bioinformatics 2004; 20: 307-315.

24. $\mathrm{Wu} \mathrm{YH}, \mathrm{Wu} \mathrm{TC}$, Liao JW, et al. p53 dysfunction by xeroderma pigmentosum group $C$ defects enhance lung adenocarcinoma metastasis via increased MMP1 expression. Cancer Res 2010; 70: 10422-10432.

25. Mahapatra S, Karnes RJ, Holmes MW, et al. Novel molecular targets of Azadirachta indica associated with inhibition of tumor growth in prostate cancer. AAPS J 2011; 13: 365-77.

26. Sullivan, JP, Spinola M, Dodge $M$, et al. Aldehyde dehydrogenase activity selects for lung adenocarcinoma stem cells dependent on notch signaling. Cancer Res 2010; 70: 9937-9948.

27. Jiang, LY, Zhang XL, Du P, Zheng JH. Gamma-secretase inhibitor, DAPT inhibits self-renewal and stemness maintenance of ovarian cancer stem-like cells in vitro. Chin J Cancer Res 2011; 23: 140-146.

28. Chiu WT, Huang YF, Tsai HY, et al. FOXM1 confers to epithelial-mesenchymal transition, stemness and chemoresistance in epithelial ovarian carcinoma cells. Oncotarget 2015; 6: 2349-2365.

29. Wu YH, Chang TH, Huang YF, Chen CC, Chou CY. COL11A1 confers chemoresistance on ovarian cancer cells through the activation of Akt/c/EBP $\beta$ pathway and PDK1 stabilization. Oncotarget 2015, Accepted.

30. Hu K, Kobayashi H, Dong A, Jing Y, Iwasaki S, Yao X. Antineoplastic agents. III: Steroidal glycosides from Solanum nigrum. Planta Med 1999; 65: 35-38.

31. Young MJ, Wu YH, Chiu WT, Weng TY, Huang YF, Chou CY. All-trans retinoic acid downregulates ALDH1-mediated stemness and inhibits tumour formation in ovarian cancer cells. Carcinogenesis 2015; accepted.

32. Li X, Zhao Y, Ji M, Liu SS, Cui M, Lou HX. Induction of actin disruption and downregulation of P-glycoprotein expression by solamargine in multidrug-resistant K562/ A02 cells. Chin Med J (Engl) 2011; 124: 2038-2044. 